\title{
Investigation on developing of a piezoresistive pressure sensor for foot plantar measurement system
}

\begin{abstract}
In this paper, fundamental theories in developing piezoresistive pressure sensor will be discussed and our work on designing a foot plantar measurement system as the application will be explained. The mathematical equations and design procedures will be elaborate while the practical application will be investigate, experimented and analyzed. Simulation results from design theory will also be included and finally the conclusion of the proposed piezoresistive pressure sensor will be discussed.
\end{abstract}

Keyword: COMSOL multiphysics; Diabetic foot ulceration; FlexiForce A201; Foot plantar measurement; Piezoresistive pressure sensor; Sensor sensitivity 